\title{
Discovery of new Milky Way star cluster candidates in the 2MASS point source catalog
}

\section{Follow-up observations of cluster candidates in the Galactic Center region ${ }^{\star}$}

\author{
J. Borissova ${ }^{1,2}$, V. D. Ivanov ${ }^{2}$, D. Minniti ${ }^{1}$, D. Geisler ${ }^{3}$, and A. W. Stephens ${ }^{4}$ \\ 1 Pontificia Universidad Católica de Chile, Facultad de Física, Departamento de Astronomía y Astrofísica, \\ Av. Vicuña Mackenna 4860, 782-0436 Macul, Santiago, Chile \\ e-mail: dante@astro.puc.cl \\ 2 European Southern Observatory, Karl-Schwarzschild-Str. 2, 85748 Garching bei Munchen, Germany \\ e-mail: [jborisso;vivanov]@eso.org \\ 3 Grupo de Astronomía, Departamento de Física, Universidad de Concepcion, Casilla 160-C, Concepcion, Chile \\ e-mail: doug@kukita.cfm.udec.cl \\ ${ }^{4}$ Princeton University Observatory, Peyton Hall, Ivy Lane, Princeton, NJ 08544-1001, USA \\ e-mail: stephens@astro.princeton.edu
}

Received 19 August 2004 / Accepted 28 December 2004

\begin{abstract}
This paper is part of a project to search the inner Milky Way for hidden massive clusters and to address the question of whether our Galaxy still forms clusters similar to the progenitors of the present-day globular clusters.

We report high angular resolution deep near-infrared imaging of 21 cluster candidates selected from the catalogues of Bica et al. (2003a, A\&A, 397, 177) and Dutra et al. (2003a, A\&A, 400, 533) in a region around the Galactic Center. These catalogues were created from visual inspection of the 2MASS images. Seven objects appear to be genuine clusters, and for these objects we present estimates of extinction, distance and in some cases age and mass.

Our estimated masses range from 1200 to $5500 M_{\odot}$. These clusters are thus significantly smaller than any Galactic globular cluster, and indicate that the formation of massive young clusters such as Arches and Quintuplet is not common in the presentday Milky Way.

The remaining 14 objects are either not clusters or cannot be classified based on our data.
\end{abstract}

Key words. Galaxy: open clusters and associations: general - infrared: stars

\section{Introduction}

Stellar clusters have long been recognized as important laboratories for astrophysical research. They provide homogeneous and well-defined samples of equidistant, coeval and chemically homogeneous stars. This has made them important tools for studies of star formation and evolution in the local Universe.

The recent development of infrared instrumentation has led to the discovery of a large number of heavily obscured star clusters in the Milky Way. Imaging surveys such as the Two Micron All Sky Survey (2MASS, Skrutskie et al. 1997) and the Deep Near Infrared Southern Sky Survey (DENIS, Epchtein et al. 1997) offer material for cluster identification. It appears that such hidden clusters are surprisingly numerous. For example, Hurt et al. (2000) found two new globular clusters: 2MASS GC01 and 2MASS GC02 and very recently Kobulnicky et al. (2004) identified on the Spitzer Space

* Based on observations collected with the $6.5 \mathrm{~m}$ Magellan Baade telescope, Las Campanas Observatory.
Telescope images from the Galactic Legacy Infrared Mid-Plane Survey Extraordinaire (GLIMPSE) a low-latitude rich star cluster named "GLIMPSE-C01". Dutra \& Bica (2000) visually inspected $5 \times 5$ degrees around the Galactic center and found 58 star cluster candidates, Dutra \& Bica (2001) searched for embedded clusters and stellar groups in the area of known nebulae and added another 42 candidates. Bica et al. (2003b) summarized the new clusters and groups into a catalog containing 276 objects. Dutra et al. (2003a) searched the Southern Milky Way and added 179 more star clusters and groups. Automated searches based on stellar density contrast were carried out by Carpenter et al. (2000), Ivanov et al. (2002, hereafter Paper I), Reyle \& Robin (2002), and Borissova et al. (2003, hereafter Paper II), and yielded 22 new cluster candidates.

The total number of new infrared-detected star clusters and stellar group candidates currently is about 500 . Yet, this valuable dataset remains relatively unmined. The first step in the analysis is to identify true clusters and then to determine their physical properties. This paper is part of a project to derive 
Table 1. Parameters of the cluster candidates and the log of observations.

\begin{tabular}{|c|c|c|c|c|c|c|c|}
\hline ID & \multicolumn{4}{|c|}{ (J2000.0) } & Filter & $\begin{array}{c}\text { Exposure } \\
\mathrm{s}\end{array}$ & $\begin{array}{c}\text { Date } \\
\text { observ. }\end{array}$ \\
\hline [DB2000] 7 & 175006.1 & -285313 & 0.54 & -0.81 & $K_{\mathrm{S}}$ & 60 & 01.08 .2003 \\
\hline [DB2000] 8 & 175004.7 & -285240 & 0.55 & -0.80 & $K_{\mathrm{S}}$ & 60 & 01.08 .2003 \\
\hline [DB2000] 26 & 174841.5 & -280142 & 1.12 & -0.10 & $J, K_{\mathrm{S}}$ & 600,600 & 01.08 .2003 \\
\hline [DB2000] 40 & 175315.9 & -264652 & 2.71 & -0.34 & $K_{\mathrm{S}}$ & 60 & 01.08 .2003 \\
\hline [DB2000] 41 & 175302.8 & -263926 & 2.84 & +0.39 & $K_{\mathrm{S}}$ & 60 & 01.08 .2003 \\
\hline [DB2000] 52 & 174228.1 & -295623 & 358.78 & +0.05 & $J, K_{\mathrm{S}}$ & 300,600 & 31.07 .2003 \\
\hline [DB2000] 56 & 174624.2 & -292219 & 359.71 & -0.37 & $K_{\mathrm{S}}$ & 50 & 02.08 .2003 \\
\hline [DB2000] 58 & 174500.1 & -285137 & 359.99 & +0.15 & $K_{\mathrm{S}}$ & 75 & 02.08 .2003 \\
\hline [DB2001] 40 & 173028.2 & -344130 & 353.42 & -0.36 & $J, K_{\mathrm{S}}$ & 300,600 & 01.08 .2003 \\
\hline [DBSB2003] 83 & 131114.0 & -624504 & 305.20 & +0.03 & $K_{\mathrm{S}}$ & 30 & 02.08 .2003 \\
\hline [DBSB2003] 84 & 131117.0 & -624546 & 305.20 & +0.02 & $K_{\mathrm{S}}$ & 30 & 02.08 .2003 \\
\hline [DBSB2003] 170 & 162858.0 & -493627 & 334.72 & -0.65 & $K_{\mathrm{S}}$ & 300 & 25.06 .2004 \\
\hline [DBSB2003] 174 & 164810.0 & -452129 & 340.05 & -0.23 & $H$ & 300 & 25.06.2004 \\
\hline [DBSB2003] 177 & 170413.0 & -422002 & 344.22 & -0.60 & $H, K_{\mathrm{S}}$ & 300,300 & 25.06 .2004 \\
\hline [DBSB2003] 179 & 171132.0 & -391038 & 347.58 & +0.11 & $J, H, K_{\mathrm{S}}$ & $300,300,300$ & 02.08 .2003 \\
\hline [BDSB2003] 9 & 181753.0 & -114426 & 18.67 & +1.97 & $K_{\mathrm{S}}$ & 150 & 02.08 .2003 \\
\hline [BDSB2003] 101 & 172534.0 & -342308 & 355.46 & -0.38 & $K_{\mathrm{S}}$ & 300 & 01.08 .2003 \\
\hline [BDSB2003] 103 & 175047.0 & -311634 & 358.57 & -2.16 & $J, K_{\mathrm{S}}$ & 150,150 & 02.08 .2003 \\
\hline [BDSB2003] 105 & 180730.0 & -254430 & 5.20 & -2.60 & $K_{\mathrm{S}}$ & 300 & 25.06 .2004 \\
\hline [DBSB2003] 106 & 165417.0 & -451730 & 340.79 & -1.02 & $H, K_{\mathrm{S}}$ & 300,300 & 25.06 .2004 \\
\hline [BDSB2003] 107 & 180042.0 & -240423 & 5.90 & -0.43 & $H, K_{\mathrm{S}}$ & 300,300 & 25.06.2004 \\
\hline
\end{tabular}

extinctions, distances, ages and total masses of these cluster candidates. In particular, we are addressing the question of whether the Milky Way is still forming massive clusters similar to the progenitors of present-day globular clusters. The best known examples of such objects are the Arches (Nagata et al. 1993; see Figer et al. 2002, for latest review) and the Quintuplet clusters (Glass et al. 1987; see also Figer et al. 1999b). Are there more "Arches" in the Galaxy?

Here we report deep $J, H$, and $K_{\mathrm{S}}$ imaging of 21 cluster candidates, selected from Bica et al. (2003a) and Dutra et al. (2003a). The majority of them are located in a $10 \times 10$ degree region around the Galactic Center. The targets were selected based on their classification in these papers, and on their resemblance to the Arches and the Quintuplet on the 2MASS images.

The next section describes the data and the third section discusses the confirmed clusters in detail. The fourth section includes comments on the spuriously identified clusters and objects whose nature cannot be determined based on the currently available dataset. The last section is a summary of the results.

\section{Observations and data reduction}

Infrared imaging observations were carried out on July 31, 2003, Aug. 1-2, 2003, and June 25, 2004, with the PANIC (Persson's Auxiliary Nasmyth Infrared Camera) near-infrared imager on the 6.5-meter Baade telescope at the Las Campanas
Observatory. The instrument uses a $1024 \times 1024 \mathrm{HgCdTe}$ Hawaii detector array. The scale is 0.125 arcsec pixel ${ }^{-1}$, giving a total field of view of $2.1 \times 2.1 \mathrm{arcmin}$. The observing log is given in Table 1.

The observing strategy was typical for near-infrared imaging: we alternated between the object and a nearby sky, accumulating 1 to 3 minutes of integration at each pointing. At both the "object" and the "sky" pointing we jittered within 20-30 arcsec to minimize the effect of cosmetic defects and cosmic rays. For each target and filter the "sky" images were median-combined to create a "super-sky" image which was subtracted from the "object" images. The "super-skies" were also used to create flats. Next, we shifted the "object" images to a common position, and combined them.

The stellar photometry of these sky-subtracted and combined images was carried out using ALLSTAR in DAOPHOT II (Stetson 1993). We considered only stars with DAOPHOT errors less than $0.2 \mathrm{mag}$. The median averaged internal photometric errors are $0.03 \pm 0.02$ for the $J, H, K_{\mathrm{S}}$ magnitudes brighter than $17 \mathrm{mag}$ and $0.07 \pm 0.04$ for the fainter stars. We also added in quadrature an additional observational uncertainty of $\sim 0.03$ mag due to the sky background variations. Finally, we replaced the brightest stars (usually with $K_{\mathrm{S}}<12 \mathrm{mag}$ ), that were saturated in our photometry, with the 2MASS measurements.

The weather conditions were nonphotometric (typical seeing 1-1.2 arcsec) during all our observing runs, forcing us to 


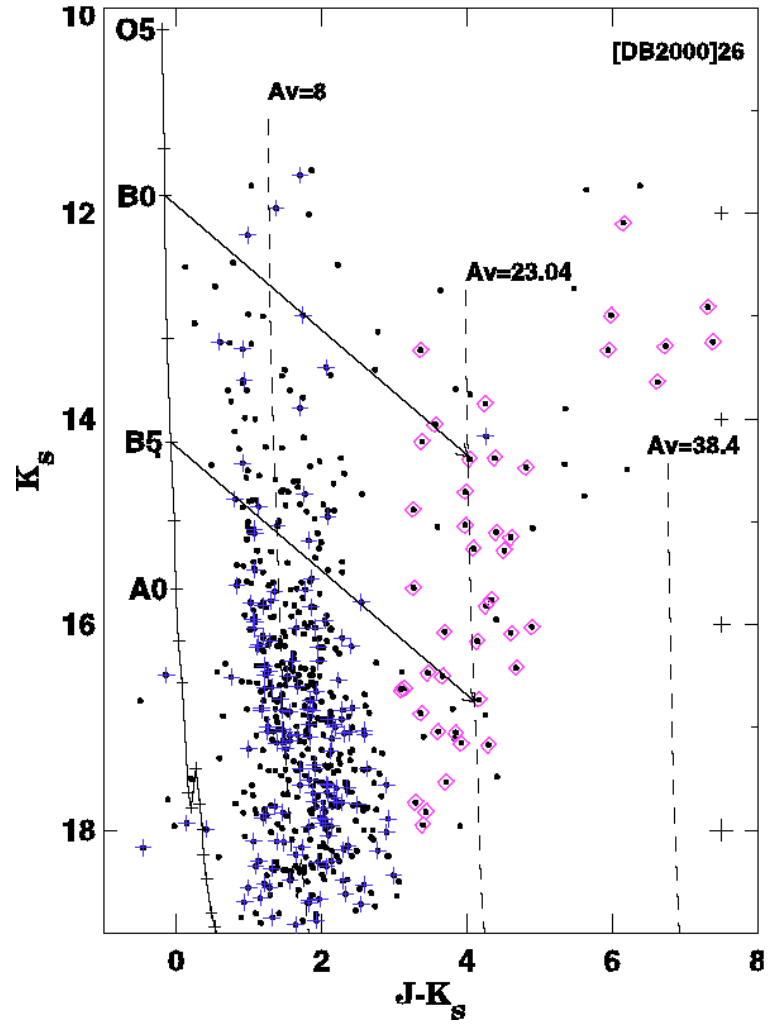

Fig. 1. The $K_{\mathrm{S}}$ versus $J-K_{\mathrm{S}}$ color-magnitude diagram of [DB2000] 26. All stars in our photometry list are shown with solid dots. The field stars are marked with pluses, and the probable cluster members with diamonds (see the text). The typical photometric errors for different magnitude bins are shown on the right. The unreddened Main Sequence (Schmidt-Kaler 1982) is shown with a solid line, and with dashed lines for different reddening values, corresponding to $E(B-V)=2.5, E(B-V)=7.2$, and $E(B-V)=12 \mathrm{mag}$. The reddening vectors for $\mathrm{B} 0 \mathrm{~V}$ and $\mathrm{B} 5 \mathrm{~V}$ stars are also shown, for $A_{\mathrm{V}}=23$ mag. The $(m-M)_{0}=15$ mag is adopted from Churchwell et al. (1990).

calibrate the data by comparing our instrumental magnitudes with the 2MASS magnitudes of 10-25 stars per image, depending on the field crowding and the band. The standard error values for the coefficients are less than 0.03 for the zeropoint and less than 0.02 for the color term. In summary, our conservative estimate of the total external errors of our photometry is 0.04-0.05 mag.

\section{Confirmed clusters}

\section{1. [DB2000] 26}

[DB2000] 26 is located on the sky close to the star-forming region $\mathrm{Sgr} \mathrm{D}$. An H II region is clearly visible on our images. The $K_{\mathrm{S}}$ versus $J-K_{\mathrm{S}}$ color-magnitude diagram is shown in Fig. 1 . If we adopt a cluster limiting radius of $r=0.5$ arcmin and plot all the stars within this area they have $J-K_{\mathrm{S}}>3$ mag. To obtain an estimate of the fore- and background contamination we define a non-cluster region in the southern part of the images with an area equal to that of the cluster. In this region we find a single "field" star that satisfies the color criterion adopted for the cluster. We then statistically clean the cluster CMD,

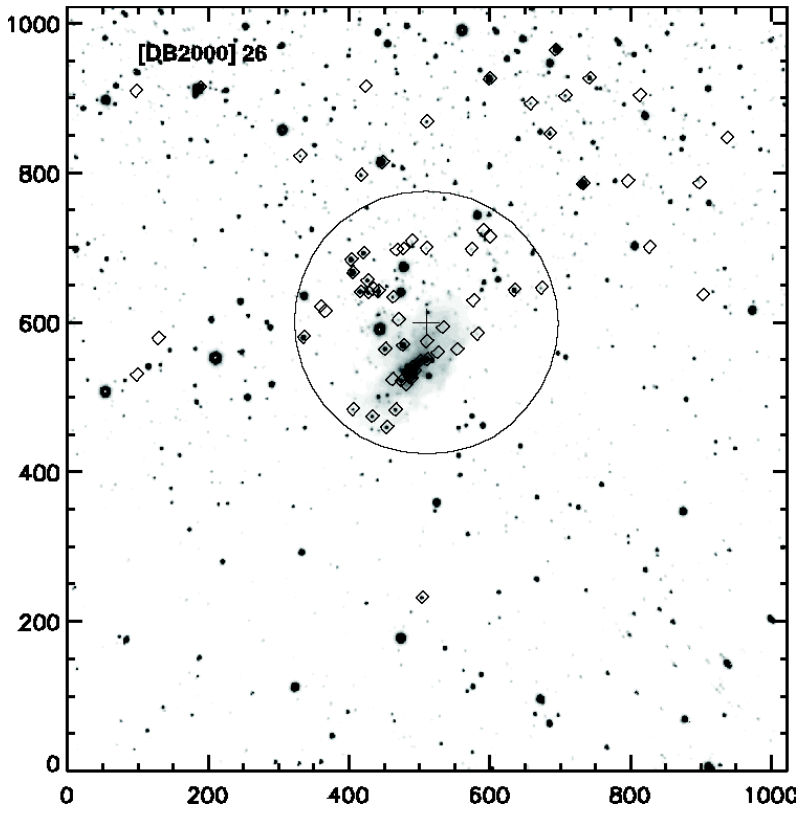

Fig. 2. The $K_{\mathrm{S}}$-band image of [DB2000] 26. The field of view is $2.1 \times 2.1 \mathrm{arcmin}$. North is up, and East is to the right. The stars with $J-K_{\mathrm{S}}>3$ mag are marked with diamonds. The large cross and the circle indicate the adopted cluster center and boundary.

removing from the cluster color-magnitude diagram as many stars as on the "field" color-magnitude diagram (in this case, one star).

To verify this selection we overploted all stars with $J-K_{\mathrm{S}}>$ 3 mag on the $K_{\mathrm{S}}$ band image (Fig. 2). The overdensity around the cluster center is obvious. We estimate that the diameter of the cluster is approximately 1.5 arcmin (shown in Fig. 2 with a large circle). The number of cluster members remaining after the "field" subtraction described above was 43 (marked in Fig. 1 with diamonds).

We estimate the extinction toward the cluster members from the $K_{\mathrm{S}}$ versus $J-K_{\mathrm{S}}$ color-magnitude diagram (Fig. 1), and adopt a distance modulus of $(m-M)_{0}=15$ mag $(D=10 \mathrm{kpc})$ based on water maser and ammonia emissions (1990). Since such sources are commonly associated with regions of recent star formation, we assumed that they originate in the vicinity of the cluster. We compared the cluster sequence with the theoretical Main Sequence from Schmidt-Kaler (1982) for $E(B-V)=2.5, E(B-V)=7.2$, and $E(B-V)=12$ mag. Throughout this paper we adopt $R_{V}=3.2$.

The cluster distance is estimated with the 10th brightest star method described in Dutra et al. (2003b; see Sect. 5 for discussion on the accuracy of this technique). Assuming that [DB2000] 26 is not a massive cluster, and that the 10th brightest star corresponds to a B0 V star with intrinsic color $(J-K)_{0}=-0.16$ and absolute magnitude $M_{k}=-3.17 \mathrm{mag}$ (Schmidt-Kaler 1982), we calculate $E(B-V)=7.0$ and $(m-M)_{0}=14.7$ mag which is good agreement with the distance determined by Churchwell et al. (1990). This is somewhat further than the distance to the star-forming region $\mathrm{Sgr} D$, placed by Blum \& Damineli (1999) close to the Galactic Center but on the near side, leaving the question about the physical association between the cluster and Sgr D open. 

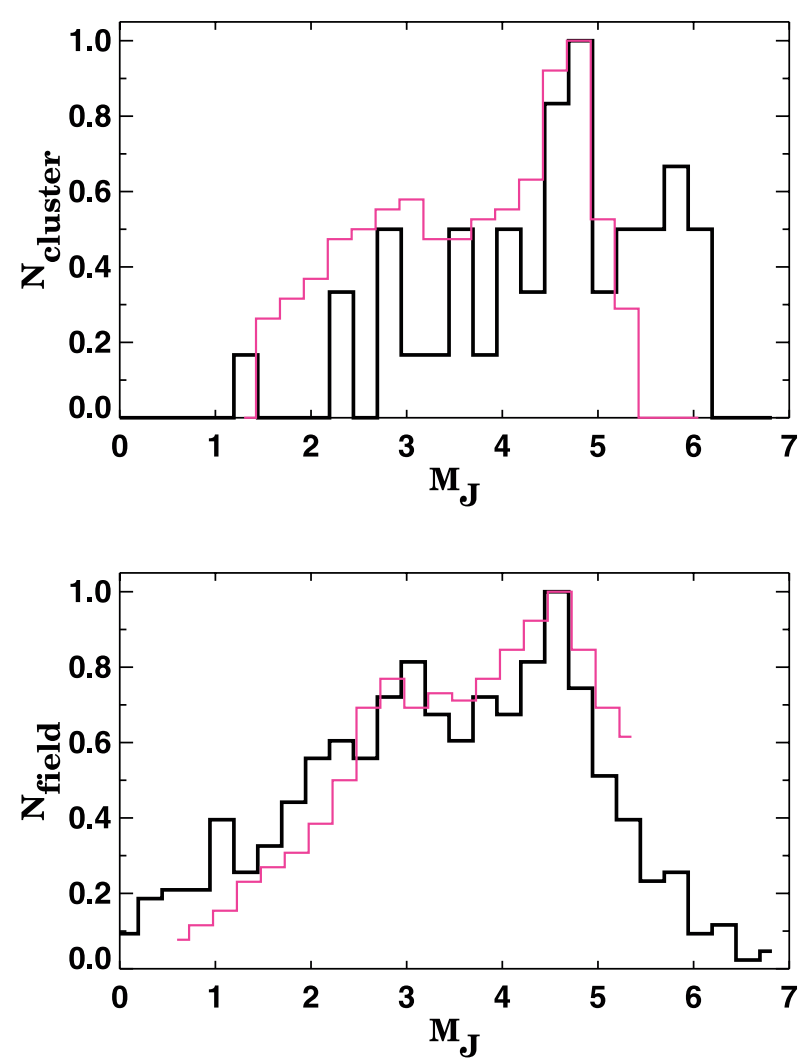

Fig. 3. The normalized luminosity function of the members of [DB2000] 26 (top), and the stars in the selected comparison field (bottom). The thick lines are the observed luminosity functions. The thin lines are the theoretical models by Strom et al. (1993) for 1 and $3 \mathrm{Myr}$, for the field and for the cluster, respectively.

The cluster members appear to be young main sequence stars. We exclude the presence of red giants as in some open clusters because the associated HII region requires the presence of ionizing photons, and red giants appear only in clusters older than 0.8 Gyr (Bertelli et al. 1994) at which time the hot stars at the upper main sequence have evolved away.

Most of these stars occupy the locus between $A_{\mathrm{V}}=18$ and 29 mag. The seven brightest stars are exception to this with $E(B-V) \sim 12 \mathrm{mag}$ or $A_{\mathrm{V}} \sim 38 \mathrm{mag}$. We adopt as a mean color excess of the cluster $E(B-V)=7.2 \mathrm{mag}$ or $A_{\mathrm{V}}=23 \mathrm{mag}$. The field stars appear to suffer between $A_{\mathrm{V}}=7$ and 12 mag of visual extinction, and for them we adopt $E(B-V)=2.8 \mathrm{mag}$ or $A_{\mathrm{V}}=9 \mathrm{mag}$.

We estimate the age of the cluster and field stars by comparing the observed and theoretical luminosity functions (LF), closely following the method described in Porras et al. (2003), based on a evolutionary sequence of six $J$-band LFs (Strom et al. 1993) for 0.3, 0.7, 1, 3, 7 and 10 Myr. We use the distance and the reddening listed above. Note that the distance to the cluster was adopted as an average distance to the field stars only for the purpose of comparing the LFs. The KolmogorovSmirnov test favors ages of $1 \mathrm{Myr}$ for the cluster and $3 \mathrm{Myr}$ for the field population with $65 \%$ and $55 \%$ respectively (not very high) probability. The model-predicted and the observed $J$-band LFs are presented in Fig. 3, where both have been normalized to a peak of one.
Finally, we attempt to determine the initial mass function slope of the cluster following the technique used in Paper II. We adopted the $1 \mathrm{Myr}$ isochrone, and counted cluster stars between reddening lines originating from positions on the isochrone for different initial masses. The photometry of the cluster members suffer severe incompleteness for stars with masses lower than 10 solar masses due to the strong extinction in the $J$-band, leaving us with two mass bins. Therefore, we can only put a limit on the IMF slope if $\Gamma \leq-0.8 \pm 0.2$. The uncertainty here represents only the formal fitting error. The minimal total cluster mass, comprised of the mass of suspected cluster members, is 780 solar masses. An integration of the extrapolated power-law IMF fit down to $0.8 M_{\odot}$ yields a total mass of $2900 M_{\odot}$ which should be considered an upper limit.

\section{2. [DB2000]52}

The cluster candidate [DB2000]52 is associated with the HII region [LPH96] 358.797+0.058, the IR source IRAS 17392-2954, four radio-sources and the young stellar object ISOGAL-P J174228.0-295614. Schultheis et al. (2003) reported $H K$-band spectroscopy of 107 sources in the region. They measured $J=13.9, K_{\mathrm{S}}=10.55$, and $A_{\mathrm{V}}=24.30 \mathrm{mag}$ for ISOGAL-P J174228.0-295614, in agreement with our photometry: $J=13.8$ and $K_{\mathrm{S}}=10.43 \mathrm{mag}$.

The cluster is located in an extremely crowded field, and while individual stellar spectra would be required to accurately determine cluster membership, statistical decontamination can nonetheless provide an estimate of the true cluster CMD and LF. Our statistical decontamination is performed as follows. First, we adopt a cluster limiting radius of $r=$ 0.5 arcmin. Next, a field with only non-cluster stars is defined as a circular annulus around the cluster with an inner radius of $r=1.1$ arcmin, and the same area as [DB2000] 52. The $\left(J-K_{\mathrm{S}}, K_{\mathrm{S}}\right)$ CMDs of the "cluster + field" and the "field" were gridded as shown in Fig. 4, and the stars in each box in the two diagrams were counted. Then, from the stars in each box of the "clusters + field" CMD we randomly removed the same number of stars as in the corresponding box of the "field" CMD. As the reddening in the field is primarily in the cluster, foreground objects will be unaffected regardless of their spatial location. Background objects are different and are assumed to contribute negligibly.

The $J-K_{\mathrm{S}}$ versus $K_{\mathrm{S}}$ color-magnitude diagram is shown in Fig. 5 and all measured stars are plotted as solid dots. The "decontaminated" cluster members are marked with diamonds. They are also shown as a diamonds on the $K_{\mathrm{S}}$ band image (Fig. 6).

We estimate the cluster distance using the 10th brightest star method (Dutra et al. 2003b), finding $E(B-V)=8.7, A_{\mathrm{V}}=$ 27.8 , and $(m-M)_{0}=11.37 \mathrm{mag}$. Assuming the extinction value obtained for ISOGAL-P J174228.0-295614 gives $(m-M)_{0}=$ $11.67 \mathrm{mag}$.

Thus, for this cluster we found reddening between $A_{\mathrm{V}}=19$ and 29 mag and several stars with infrared excess. The cluster stars are young main sequence stars. We adopted the average distance modulus $(m-M)_{0}=11.5 \mathrm{mag}$ or $D \sim 2 \mathrm{kpc}$. 

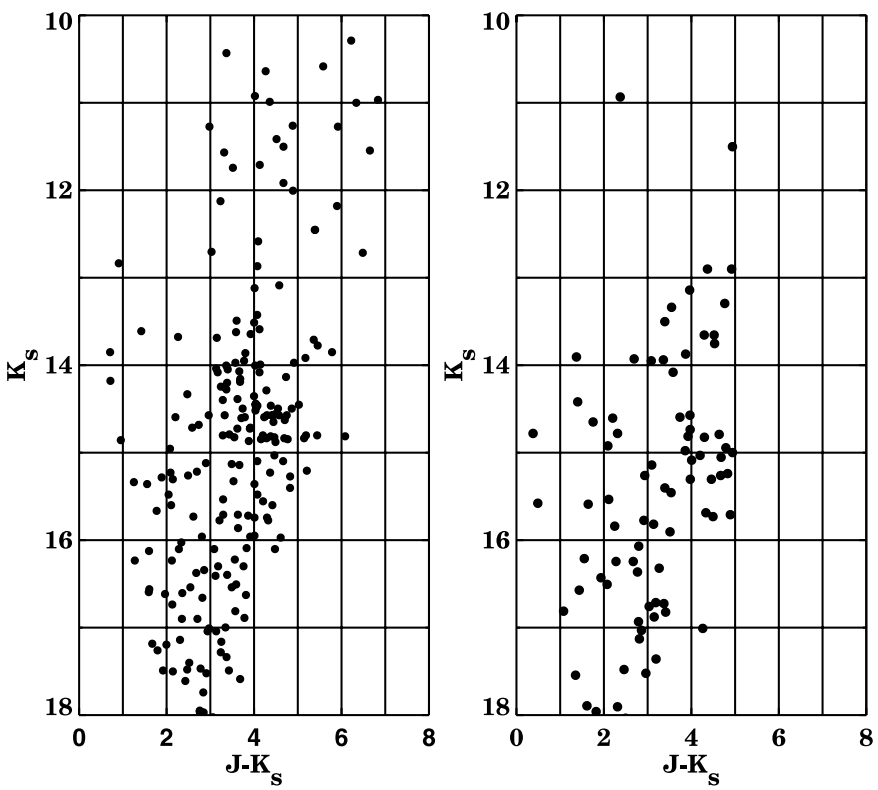

Fig. 4. The $\left(J-K_{\mathrm{S}}, K_{\mathrm{S}}\right)$ color-magnitude diagram for [DB2000] 52: "cluster + field" on the left and "field" on the right. The grid for the CMD decontamination is shown (see the text for details).

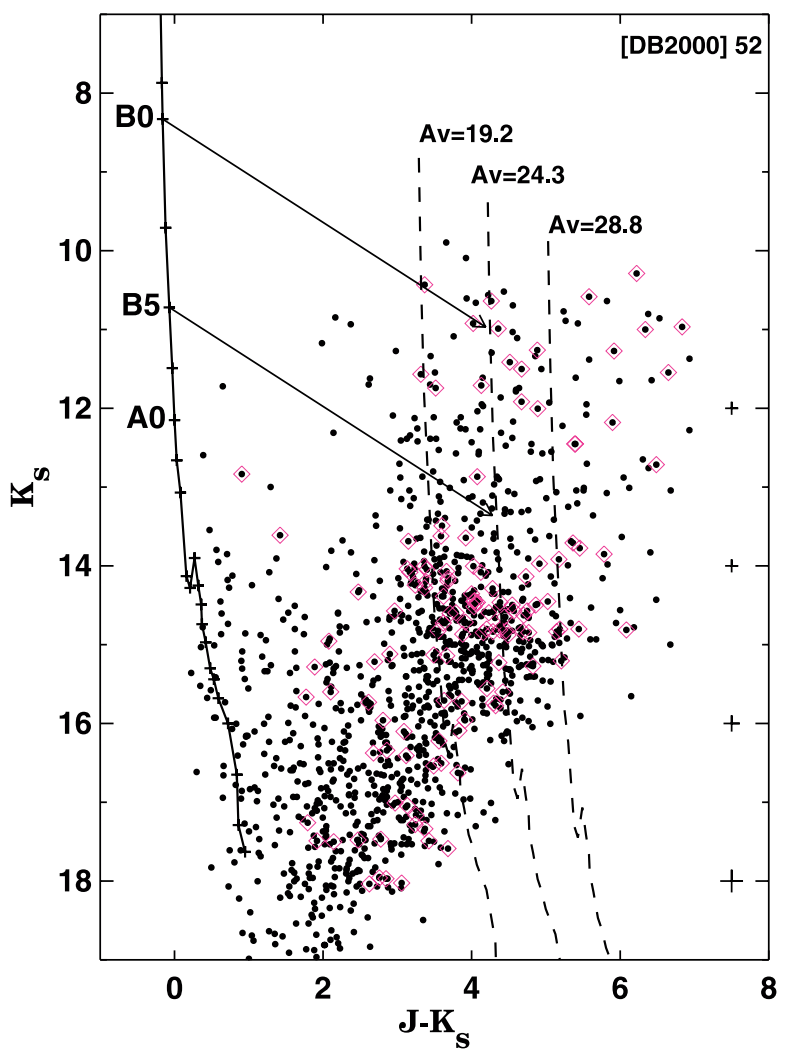

Fig. 5. The $K_{\mathrm{S}}$ versus $J-K_{\mathrm{S}}$ color-magnitude diagram of [DB2000] 52. All stars in our photometry list are shown with solid dots. The statistically decontaminated cluster members are marked with diamonds. The typical photometric errors for different magnitude bins are shown on the right. The unreddened Main Sequence (Schmidt-Kaler 1982) is shown with the solid line, and with dashed lines for different reddening values, corresponding to $E(B-V)=6$, $E(B-V)=7.6$ and $E(B-V)=9$ mag. The reddening vectors for $\mathrm{B} 0 \mathrm{~V}$ and $\mathrm{B} 5 \mathrm{~V}$ are also shown for $A_{\mathrm{V}}=23 \mathrm{mag}$. The adopted distance modulus is $(m-M)_{0}=11.5 \mathrm{mag}$.

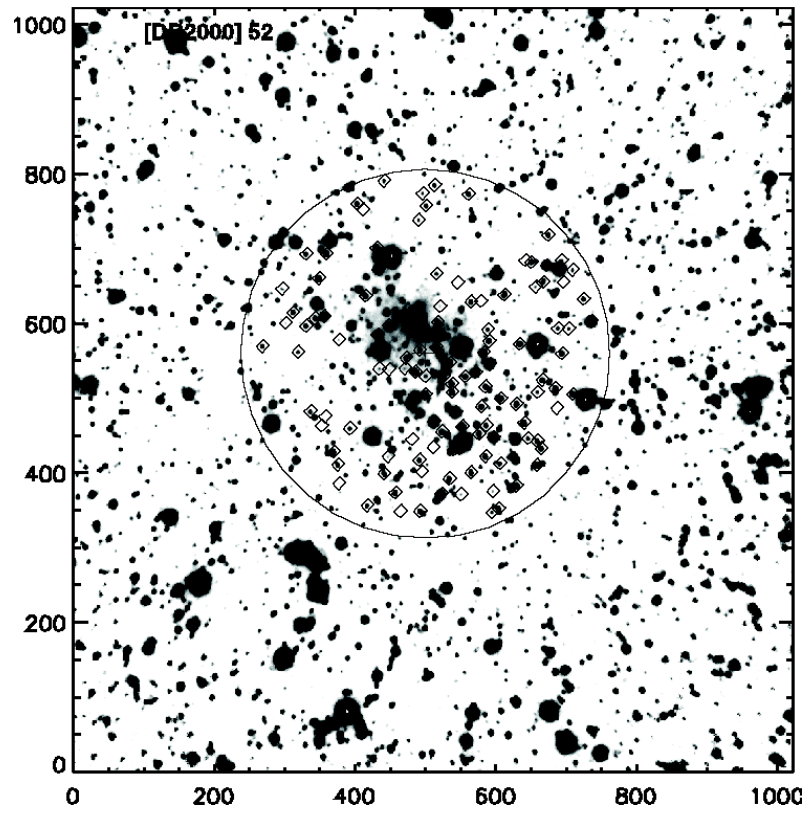

Fig. 6. The $K_{\mathrm{S}}$ band image of [DB2000] 52. The field of view is $2.1 \times 2.1 \mathrm{arcmin}$. North is up, and East is to the right. The statistically decontaminated cluster stars are marked with diamonds. The large cross and the circle indicate the adopted cluster center and boundary.

The heavy background and foreground contamination and the uncertain distance prevent us from estimating the age and the IMF slope of this cluster. The minimum total mass, comprised of suspected member stars, is $600-700 M_{\odot}$, similar to the previous object.

An upper limit to the cluster mass can be estimated by adopting the Salpeter law, normalizing it to the upper three mass bins (encompassing stars with masses about 10 solar masses), and integrating down to 0.8 solar masses. This yields a total cluster mass of $5000 M_{\odot}$ or less. This is a very conservative limit because about $20 \%$ of this mass is below 1 solar mass, and the IMF is known to flatten in this range. It is impossible to derive the IMF slope because only the two upper mass bins are complete in the $J$-band due to the extreme extinction; we therefore assume the Salpeter slope.

\section{3. [DB2001] 40 or [BDB2003] G353.42-00.36G353.42-00.36}

[DB2001]40 appears to be associated with the HII region [FC2000] G353.41-0.36. The Southern part of our images is free of nebulosity and we adopted it as a comparison field. The $K_{\mathrm{S}}$ versus $J-K_{\mathrm{S}}$ color-magnitude diagram is shown in Fig. 7, and all measured stars are plotted as solid dots. The stars from our "comparison" field are plotted with pluses. As can be seen, most of them form a sequence with $J-K_{\mathrm{S}}<3$ mag.

We marked all stars which have $J-K_{S}>3$ mag on the $K_{\mathrm{S}}$-band image (Fig. 8). An overdensity of stars around the cluster center is obvious. We estimated the cluster diameter as $\sim 0.6-0.7$ arcmin (shown in Fig. 8 with a large circle). There are 13 suspected cluster members left after the CMD decontamination. They are marked with diamonds in Fig. 7. 


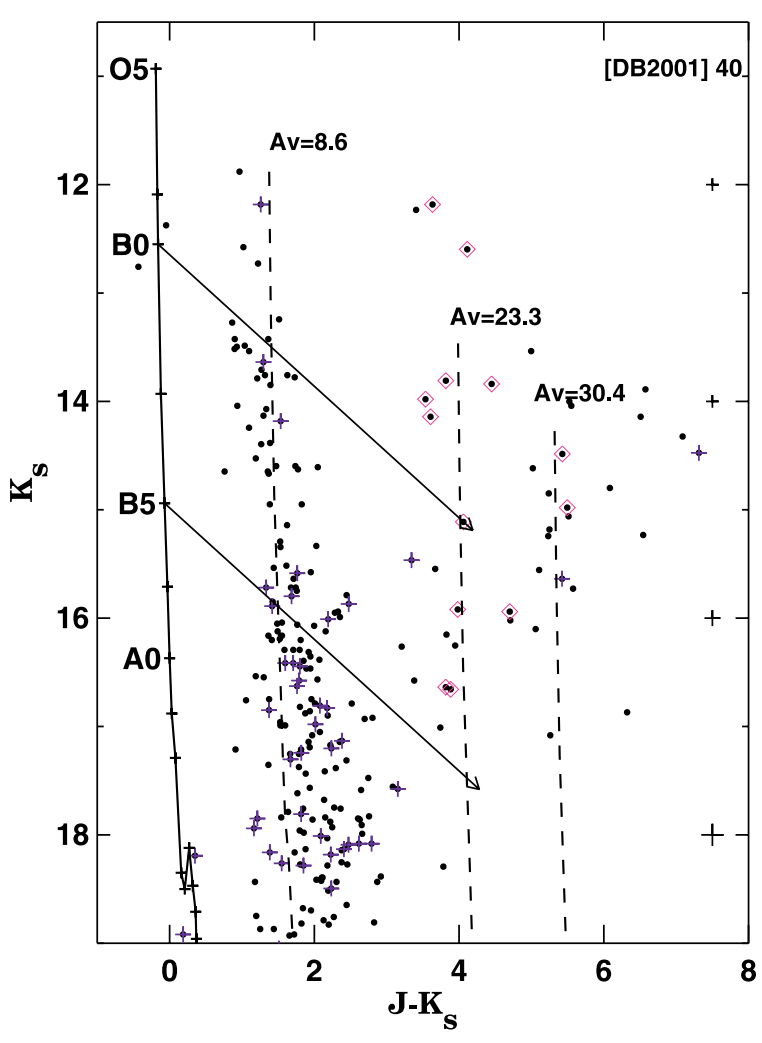

Fig. 7. The $K_{\mathrm{S}}$ versus $J-K_{\mathrm{S}}$ color-magnitude diagram of [DB2001] 40. All stars in our photometry list are shown with solid dots. The statistically decontaminated cluster members are marked with diamonds, and field stars are marked with +'s. The typical photometric errors for different magnitude bins are shown on the right. The unreddened Main Sequence (Schmidt-Kaler 1982) is shown with the solid line, and with dashed lines for different reddening values, corresponding to $E(B-V)=2.7, E(B-V)=7.2$ and $E(B-V)=9.5$ mag. The reddening vectors for $\mathrm{B} 0 \mathrm{~V}$ and $\mathrm{B} 5 \mathrm{~V}$ stars are also shown, for $A_{\mathrm{V}}=24 \mathrm{mag}$. The adopted distance modulus is $(m-M)_{0}=15.7 \mathrm{mag}$.

Here again we are forced to apply the 10th brightest star method to obtain an approximate distance estimate. It appears that [DB2001] 40 is not a massive cluster, and following Dutra et al. (2003b) we assume that the 10th brightest star is a B0 V star. From the difference between the apparent and the intrinsic color and the apparent and the intrinsic magnitude we derive $E(B-V)=7.3, A_{\mathrm{V}}=23.4$ and $(m-M)_{0}=15.7 \mathrm{mag}$ $(D=13.8 \mathrm{kpc})$. This distance is in good agreement with that from Walsh et al. (1997), who obtained a kinematical distance of $15.6 \mathrm{kpc}$ for IRAS $17271-3439$. In their study of ultracompact (UC) HII regions they derived the distances kinematically, using the $6.669 \mathrm{GHz}$ methanol maser emission velocity. The small number of cluster members renders any IMF slope derivation meaningless. The observed members comprise a total mass of about 400 solar masses. The upper mass limit of this cluster is 450 solar masses but it is more uncertain in comparison with the other confirmed clusters because of the small number of potential cluster members.

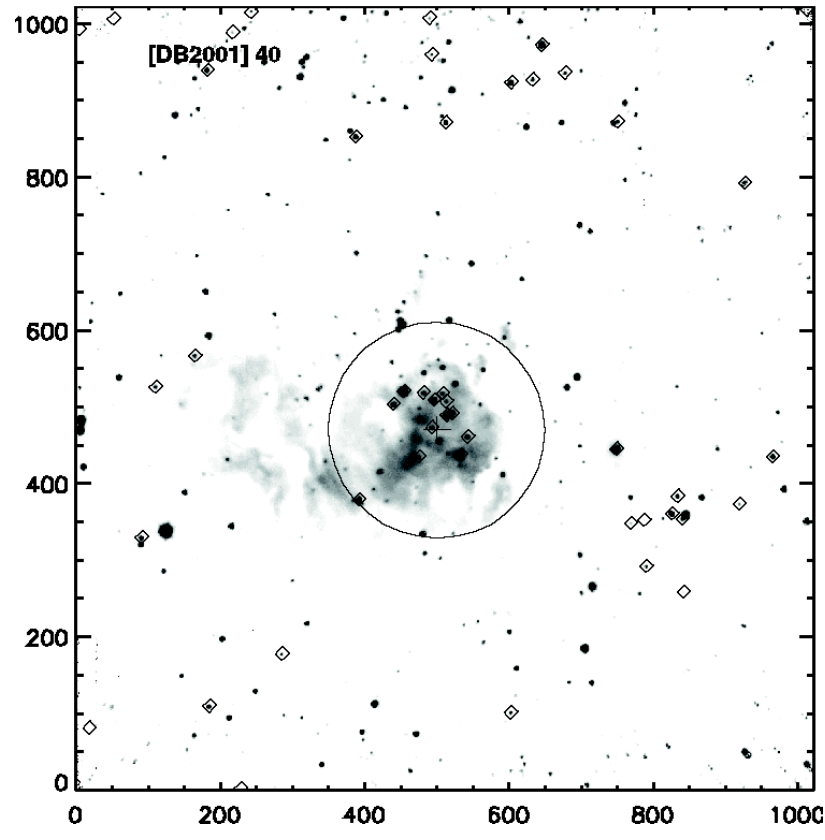

Fig. 8. The $K_{\mathrm{S}}$ band image of [DB2001] 40 . The field of view is $2.1 \times$ 2.1 sarcmin. North is down, and East is to the left. The stars with $J-K_{\mathrm{S}}>3$ mag are marked with diamonds. The large cross and the circle indicate the adopted cluster center and boundary.

\section{4. [DBSB2003] 177}

Walsh et al. (1997) found an ultra-compact H II region in the vicinity of [DBSB2003] 177. The region is associated with a red star $\left(J-K_{\mathrm{S}}=4.7\right)$. They detected a methanol and $\mathrm{OH}$ maser source 5 arcsec to the North, with no apparent near-infrared counterpart.

To decontaminate the cluster's CMD we followed the same method as for [DB2000] 52, adopting a cluster limiting radius of $r=0.5$ arcmin. The "field" population was determined from a circular annulus around the cluster, with an inner radius $r=1.1$ arcmin and the same area as the cluster. The gridded $\left(H-K_{\mathrm{S}}, K_{\mathrm{S}}\right)$ CMDs of "cluster + field" and "field" are shown in Fig. 9.

The $K_{\mathrm{S}}$ versus $H-K_{\mathrm{S}}$ color-magnitude diagram of all stars in the images is shown in Fig. 10. The 23 suspected members remaining after the "decontamination" are marked with diamonds. Their spatial location (again, marked with diamonds) is shown on the $K_{\mathrm{S}}$ band image in Fig. 11 .

Again, we use the 10th brightest star method to estimate the cluster distance, under the assumption that [DBSB2003] 177 is not a massive cluster and the 10th brightest star corresponds to a B0 V star with $(H-K)_{0}=-0.04$ and $M_{K}=-3.17 \mathrm{mag}$ (Schmidt-Kaler 1982). We obtain $E(B-V)=5, A_{\mathrm{V}}=16$ and $(m-M)_{0}=16.3 \mathrm{mag}(18 \mathrm{kpc})$. This distance is close to the kinematical distance of $16.6 \mathrm{kpc}$ towards IRAS 17006-4215, located in the vicinity of the cluster (Walsh et al. 1997). Assuming an age of $1 \mathrm{Myr}$, the suspected cluster members comprise $200-400 M_{\odot}$. As before, we refrain from further analysis because of the uncertain distance. The upper mass limit for this cluster is $1200 M_{\odot}$, based on an integration of the LF down to $0.8 M_{\odot}$, assuming a Salpeter slope. 

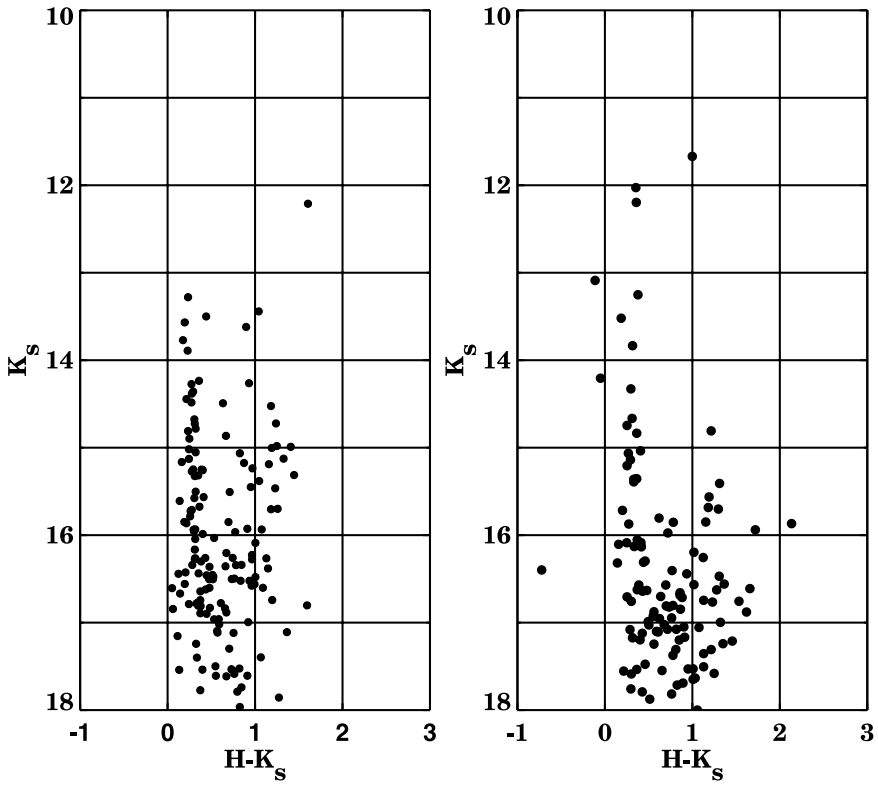

Fig. 9. The $\left(H-K_{\mathrm{S}}, K_{\mathrm{S}}\right)$ color-magnitude diagram for [DBSB2003] 177: "cluster + field" on the left and "field" on the right. The grid for the CMD decontamination is shown (see the text for details).

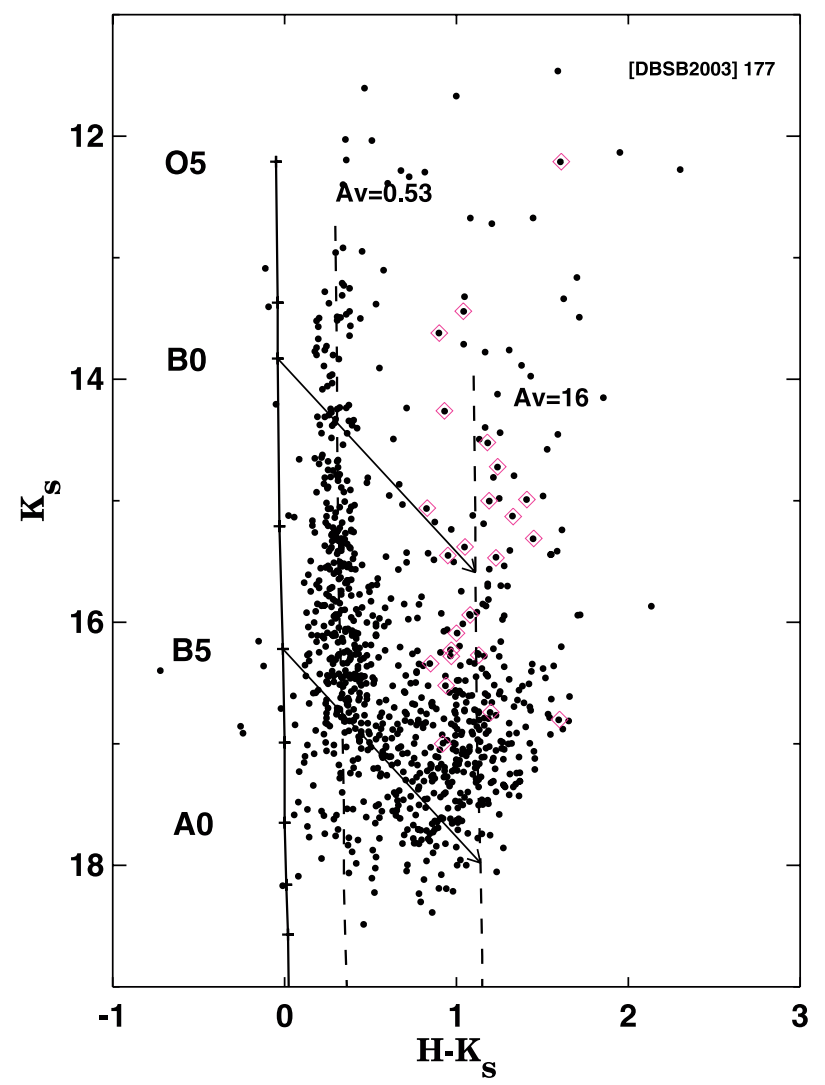

Fig. 10. The $K_{\mathrm{S}}$ versus $H-K_{\mathrm{S}}$ color-magnitude diagram of [DBSB2003] 177. All stars in our photometry list are shown with solid dots. The decontaminated cluster members are marked with diamonds. The unreddened Main Sequence (Schmidt-Kaler 1982) is shown with solid line, and with dashed lines for different reddening values, corresponding to $E(B-V)=1.5$, and $E(B-V)=5 \mathrm{mag}$. The reddening vectors for $\mathrm{B} 0 \mathrm{~V}$ and $\mathrm{B} 5 \mathrm{~V}$ stars are also shown, for $A_{\mathrm{V}}=19 \mathrm{mag}$. The adopted distance modulus is $(m-M)_{0}=17 \mathrm{mag}$.

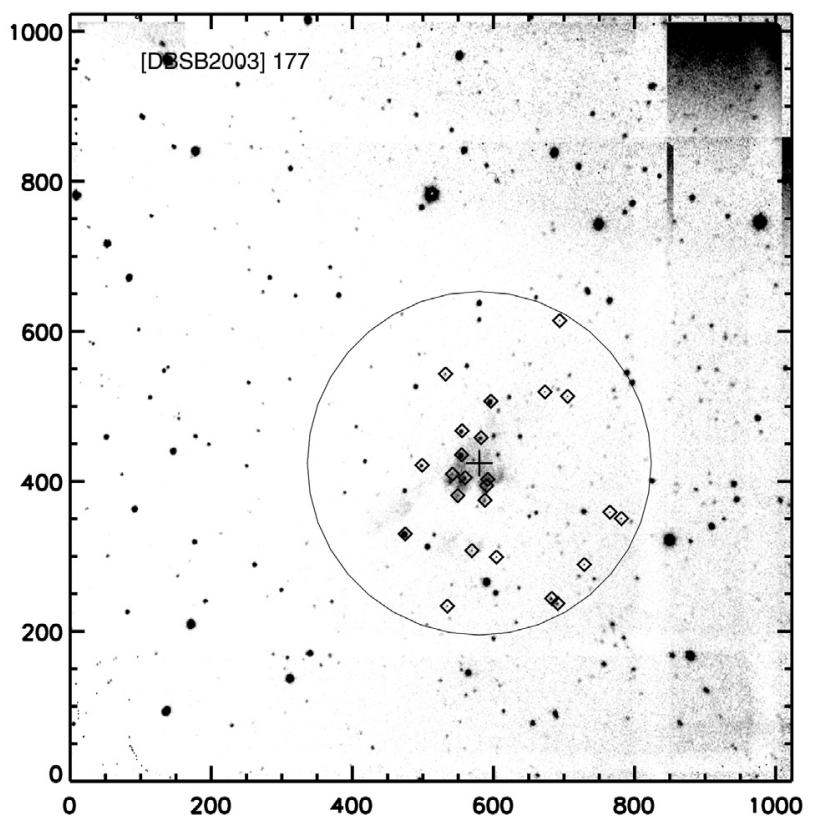

Fig. 11. The $K_{\mathrm{S}}$ band image of [DBSB2003] 177. The field of view is $2.1 \times 2.1 \mathrm{arcmin}$. North is down, and East is to the left. The statistically decontaminated cluster stars are marked with diamonds. The large cross and the circle indicate the adopted cluster center and boundary.

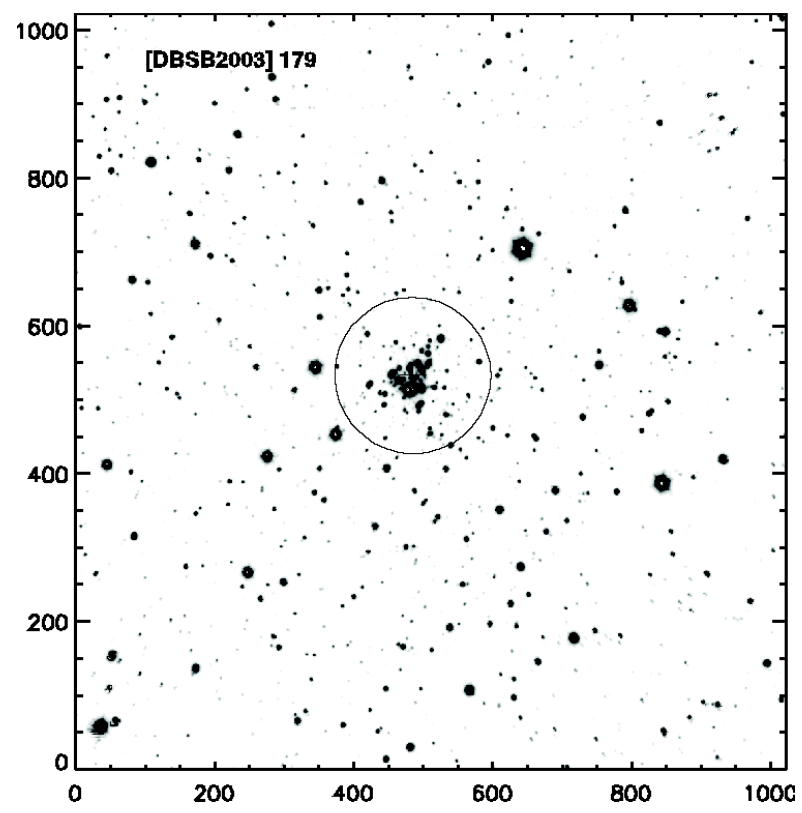

Fig. 12. The $K_{\mathrm{S}}$ band image of [DBSB2003] 179. The field of view is $2.1 \times 2.1$ arcmin. North is down, and East is to the left. The large cross and the circle indicate the adopted cluster center and boundary.

\section{5. [DBSB2003] 179}

A $K_{\mathrm{S}}$-band image of the candidate is shown in Fig. 12. The overdensity of stars is obvious.

The decontamination procedure described previously left 152 potential cluster members within 0.5 arcmin of the cluster center. The $K_{\mathrm{S}}$ versus $J-K_{\mathrm{S}}$ color-magnitude diagram is plotted in Fig. 13. 


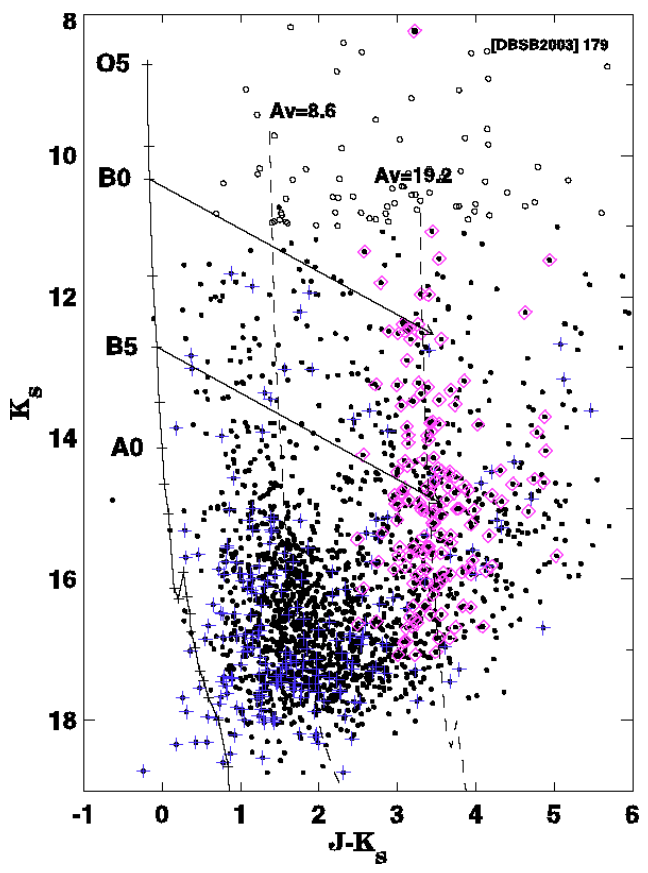

Fig. 13. The $K_{\mathrm{S}}$ versus $H-K_{\mathrm{S}}$ color-magnitude diagram of [DBSB2003] 179. All stars in our photometry list are shown with solid dots. The statistically decontaminated cluster members are marked with diamonds, field stars are marked with +'s, and open circles are for bright stars added from 2MASS photometry. The unreddened Main Sequence (Schmidt-Kaler 1982) is shown with the solid line, and with dashed lines for different reddening values, corresponding to $E(B-V)=2.7$, and $E(B-V)=6 \mathrm{mag}$. The reddening vectors for $\mathrm{B} 0 \mathrm{~V}$ and $\mathrm{B} 5 \mathrm{~V}$ stars are also shown, for $A_{\mathrm{V}}=20 \mathrm{mag}$. The adopted distance modulus is $(m-M)_{0}=13.5 \mathrm{mag}$.

The 10th brightest star method yields $E(B-V)=6$, $A_{\mathrm{V}}=19$ and $(m-M)_{0}=13.5 \mathrm{mag}(5 \mathrm{kpc})$. With such obtained parameters, a comparison with the theoretical luminosity functions of Strom et al. (1993) gives an age of the cluster 7-8 Myr. Predicted and observed $J$ band LFs are presented in Fig. 14.

Given these parameters, the total mass of the cluster members is $1100-1900 M_{\odot}$. The upper mass limit for this cluster is $5500 M_{\odot}$.

\section{6. [DBSB2003] 106}

The $K_{\mathrm{S}}$-band image of [DBSB2003] 106 is shown in Fig. 15. There is an obvious stellar overdensity in the center of the field, associated with extended emission from a gaseous nebulosity.

We followed the same analysis procedure as for the previous objects, and after the decontamination we selected 152 possible cluster members within 0.5 arcmin from the cluster center. The $K_{\mathrm{S}}$ versus $H-K_{\mathrm{S}}$ color-magnitude diagram is shown in Fig. 16.

The 10th brightest star method gives $E(B-V)=6.5$, $A_{\mathrm{V}}=20.8$, and $(m-M)_{0}=15.2 \mathrm{mag}(11 \mathrm{kpc})$. Again, we refrained from further analysis but the presence of the emission nebula around the cluster indicates that it is $5 \mathrm{Myr}$ of age or younger. The mass of the detected cluster members is 600-800 solar masses. The upper mass limit for this cluster is 4000 solar masses.

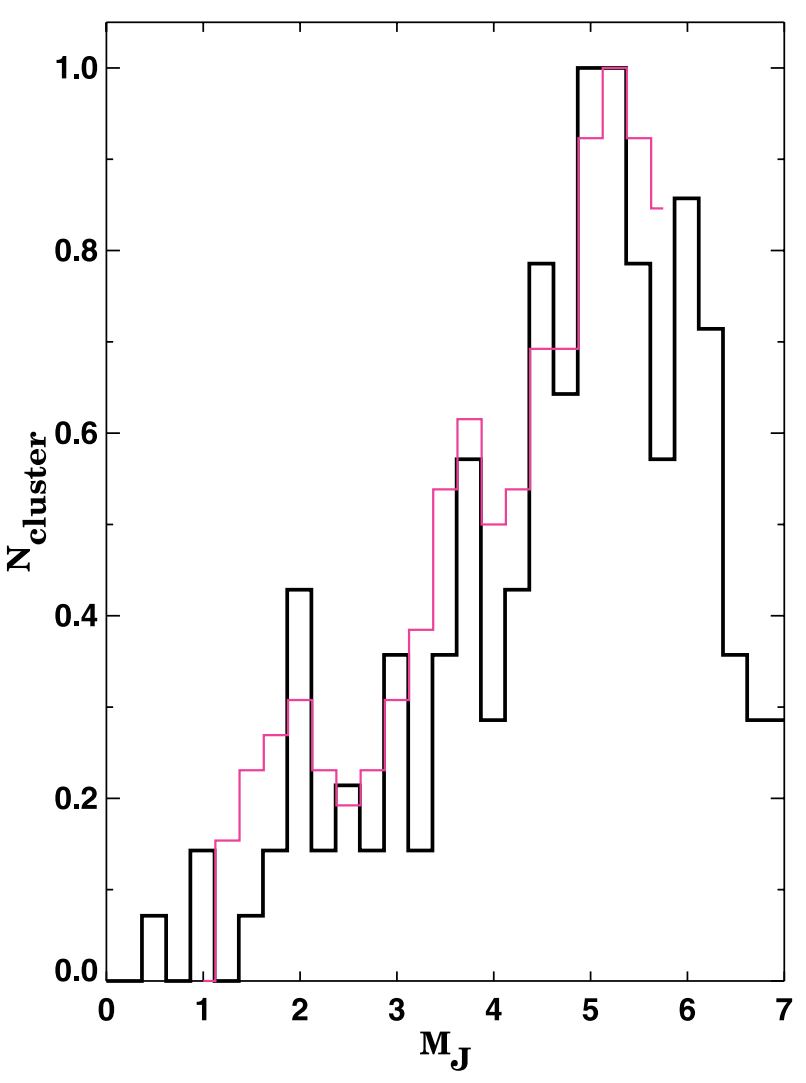

Fig. 14. The normalized luminosity function of the [DBSB2003] 179 cluster (thick line), and the theoretical $7 \mathrm{Myr}$ model by Strom et al. (1993) (thin line).

\section{7. [BDSB2003] 107}

The $K_{\mathrm{S}}$-band image of [BDSB2003] 107 is shown in Fig. 17. This is the largest cluster candidate in our sample, and here we adopted a radius of $1 \mathrm{arcmin}$. The object is probably associated with the HII region WC89005.09-0.39A, so the object must be younger than $5 \mathrm{Myr}$.

The CMD decontamination leaves 309 possible cluster members. The $K_{\mathrm{S}}$ versus $H-K_{\mathrm{S}}$ color-magnitude diagram is shown in Fig. 18. The 10th brightest star method gives $E(B-V)=5.7, A_{\mathrm{V}}=18.2$, and $(m-M)_{0}=13.8 \mathrm{mag}$ $(5.8 \mathrm{kpc})$. The total mass of the measured cluster members is 800-1000 solar masses. The upper mass limit for this cluster is 4500 solar masses.

\section{Spurious candidates and uncertain detections}

Our data reveal that some candidates are actually not clusters. Most often they turned out to be just one or more bright stars, well above the 2MASS saturation limit. Located close enough, such stars closely resemble compact clusters at the 2MASS resolution. We consider groups of up to a dozen bright stars not to be clusters unless a concentration of some fainter stars is also observable. In this section we also list objects for which our data does not allow us to derive a definitive conclusion. A mosaic of our cluster images is shown in Fig. 19. In addition, one of the cluster candidates appeared to be a well-known globular cluster. 


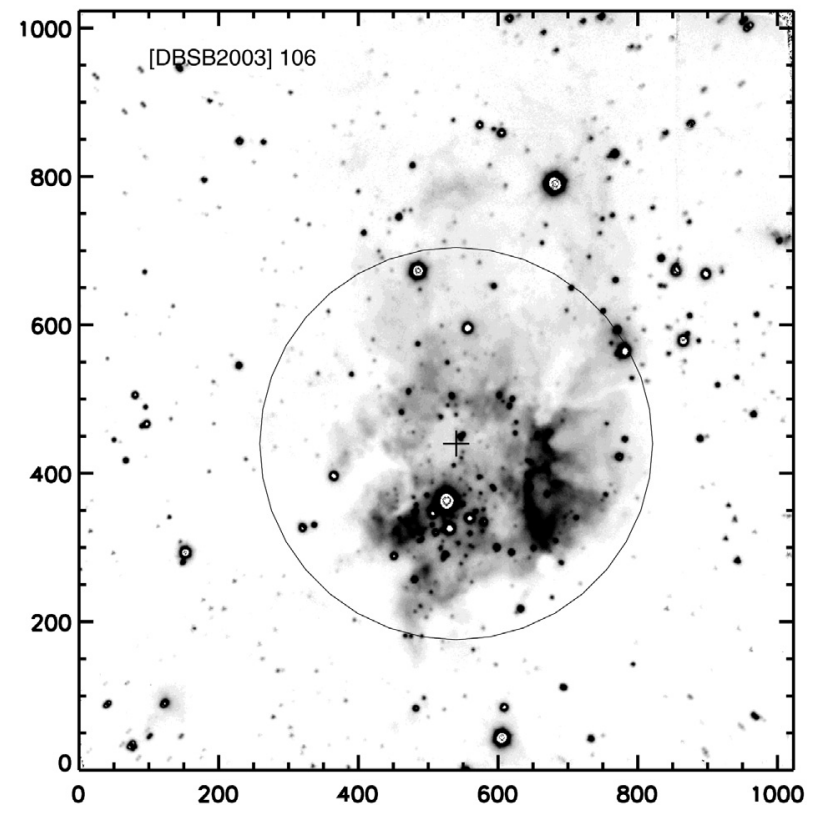

Fig. 15. The $K_{\mathrm{S}}$ band image of [DBSB2003] 106. The field of view is $2.1 \times 2.1 \mathrm{arcmin}$. North is down, and East is to the left. The large cross and the circle indicate the adopted cluster center and boundary.

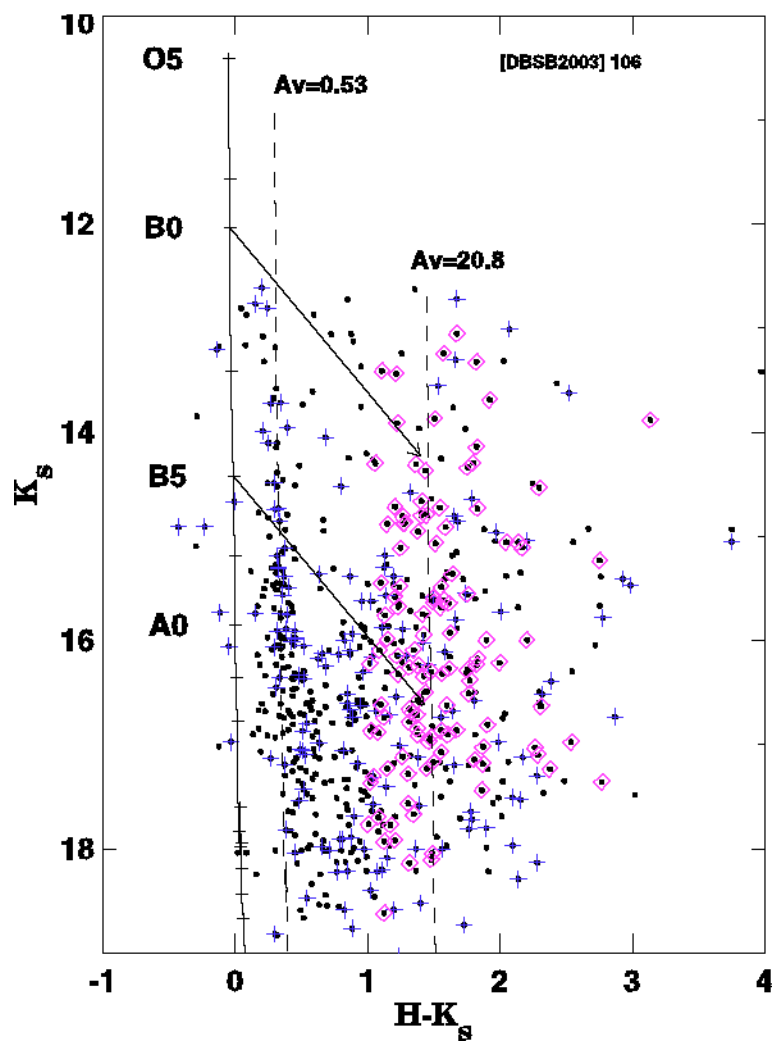

Fig. 16. The $K_{\mathrm{S}}$ versus $H-K_{\mathrm{S}}$ color-magnitude diagram of [DBSB2003] 106. All stars in our photometry list are shown with solid dots. The statistically decontaminated cluster members are marked with diamonds. The unreddened Main Sequence (Schmidt-Kaler 1982 ) is shown with the solid line, and with dashed lines for different reddening values, corresponding to $E(B-V)=1.5$, and $E(B-V)=6.5$ mag. The reddening vectors for B0 V and B5 V stars are also shown, for $A_{\mathrm{V}}=20 \mathrm{mag}$. The adopted distance modulus is $(m-M)_{0}=15.2 \mathrm{mag}$.

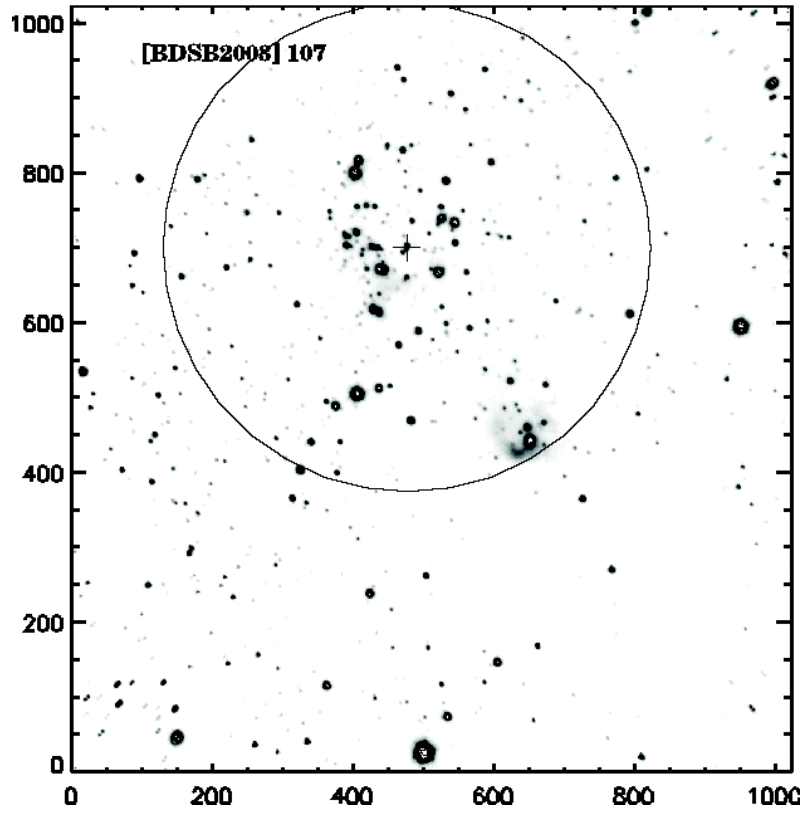

Fig. 17. The $K_{S}$ band image of [BDSB2003] 107. The field of view is $2.1 \times 2.1 \mathrm{arcmin}$. North is up, and East is to the right. The large cross and the circle indicate the adopted cluster center and boundary.

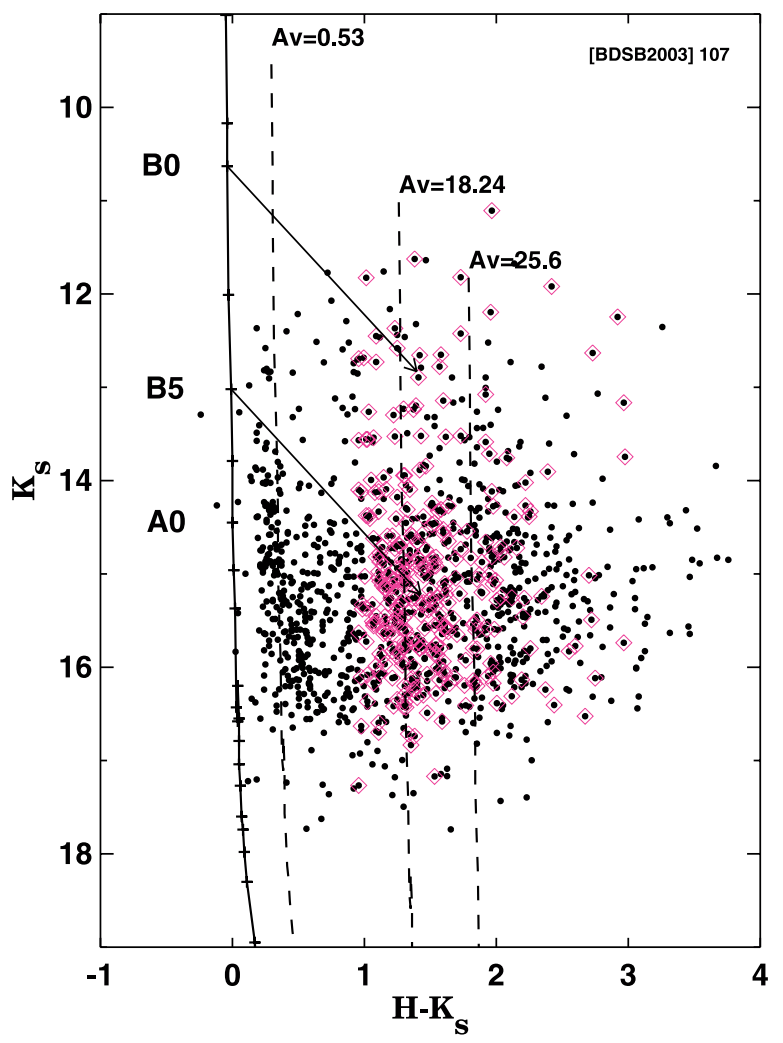

Fig. 18. The $K_{\mathrm{S}}$ versus $H-K_{\mathrm{S}}$ color-magnitude diagram of [BDSB2003] 107. All stars in our photometry list are shown with solid dots. The decontaminated cluster members are marked with diamonds. The unreddened Main Sequence (Schmidt-Kaler 1982) is shown with solid line, and with dashed lines for different reddening values, corresponding to $E(B-V)=1.5$, and $E(B-V)=5.7 \mathrm{mag}$. The reddening vectors for $\mathrm{B} 0 \mathrm{~V}$ and $\mathrm{B} 5 \mathrm{~V}$ are also shown, for $A_{\mathrm{V}}=20 \mathrm{mag}$. The adopted distance modulus is $(m-M)_{0}=13.8 \mathrm{mag}$. 


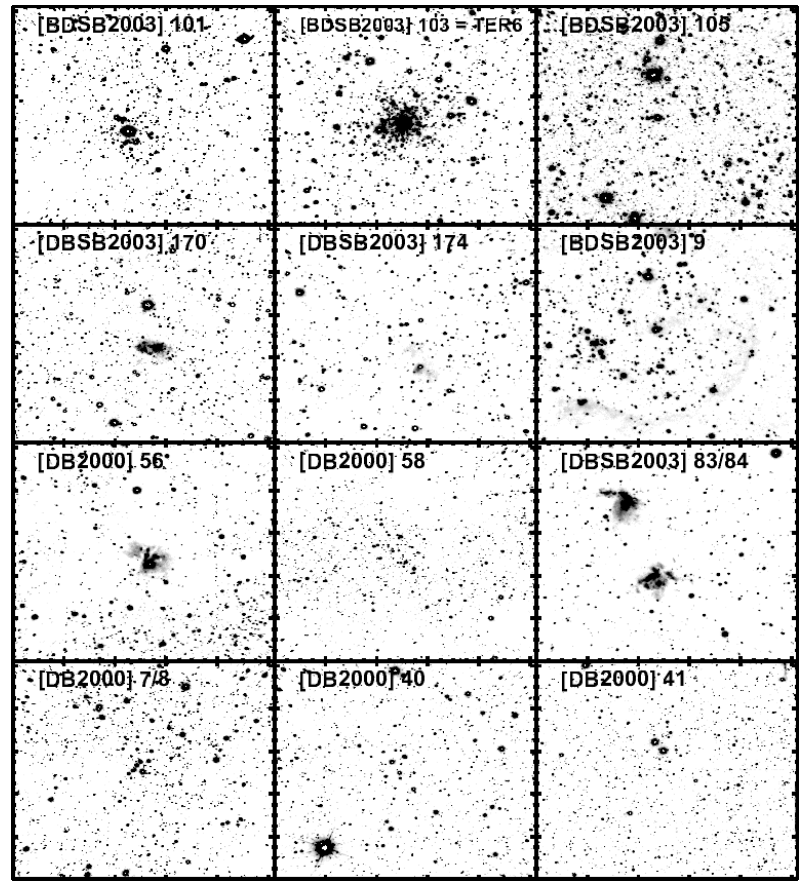

Fig. 19. Mosaic $K_{\mathrm{S}}$-band images of the spurious clusters and objects with uncertain nature. The images are 2.1 arcmin on the side.

1. [DB2000]7 and [DB2000] 8: on our 160 -s $K_{\mathrm{S}}$-band image the cluster candidate [DB2000]7 is resolved into $7-8$ bright stars. There is no overdensity of fainter stars close to the center of the object in comparison with the periphery of the image. There are no associated H II regions or IRAS sources, unlike the typical young clusters. The same field contains the cluster candidate [DB2000] 8, - also resolved into several bright stars, but showing no overdensity of fainter stars. The cluster-like appearance of [DB2000] 7 and [DB2000] 8 on the 2MASS images is likely due to the limited angular resolution.

2. [DB2000]40: based on one 60-s $K_{\mathrm{S}}$-band image, the cluster candidate [DB2000] 40 appears to be a group of 6-7 bright stars. There is no overdensity of fainter stars, and no associated $\mathrm{H}$ II regions or an IRAS source.

3. [DB2000] 41 was resolved into two bright stars on a 60-s $K_{\mathrm{S}}$-band image.

4. [DB2000] 56: Dutra et al. (2003a) classifies this object as deeply embedded in dust and gas. Our data (50-s $K_{\mathrm{S}}$-band image) shows that this is one bright star in the ultacompact H II region.

5. [DB2000]58: Dutra et al. (2003) report that this cluster is related to the optical H II region Sh 2-17. Our 75-s $K_{\mathrm{S}}$-band image indeed shows a conglomerate of about a dozen bright stars, but no overdensity of fainter stars.

6. [DBSB2003] 83 and [DB2000] 84: both objects were resolved into single bright stars, embedded in an ultracompact $\mathrm{H}$ II region.

7. [DBSB2003] 170 is an ultracompact H II region, which contains several bright stars. No concentration of faint stars is present.

8. [DBSB2003] 174 is a group of bright stars associated with an $\mathrm{H}$ II region, but there is no overdensity of faint stars.
9. [BDSB2003] 9 shows a concentration of stars, associated with a H II region. The object has the appearance of a star forming region but we have only a single 150 -s $K_{\mathrm{S}}$-band image, preventing us from carrying out further analysis. This object needs further observations.

10. [BDSB2003] 101 is resolved into two bright stars on our 300 -s $K_{\mathrm{S}}$-band image.

11. [BDSB2003] 103 is the well-known globular cluster Terzan 6.

12. [BDSB2003] 105 is a single bright star.

\section{Uncertainties in the distance estimates}

The 10th brightest star method described in Dutra et al. (2003b) was used to determine the cluster distance. This method is meant to improve statistics when estimating the distance of a cluster. Rather than just looking at the magnitude of the brightest star, which is very uncertain due to the small number of stars, they look farther down the MS where there are more stars, and hence the difference between the nth and $(n+1)$ th star is much smaller. One would like to look at the entire population, or at least the entire upper MS as a whole to get the best results. This technique gives only an approximate estimate, with systematic and random errors.

The former ones can be estimated internally by comparison between the distances derived from the assumptions that the cluster is massive versus non-massive, which corresponds to the 10th star being $\mathrm{O} 5 \mathrm{~V}$ or B0 V, respectively. This leads to a difference in the distance modulus of $\sim 1.5 \mathrm{mag}$, or factor of $\sim 4$. Externally, the uncertainty can be estimated from comparison with distances obtained from other techniques, which are not available for the majority of the objects.

The random errors can be quantified by assuming Poisson statistics in the number of stars. As was mentioned above, the method assumes that the 10th brightest stars is a B0 V. Restating this, there are $10 \pm 3$ stars brighter than the $\mathrm{B} 0 \mathrm{~V}$ star in the cluster. Therefore, we assumed that the $1 \sigma$ random error is the difference between the extinction and distance values derived assigning the $\mathrm{B} 0 \mathrm{~V}$ spectral type to the 10th brightest star, and to the 13th star. In effect, here we simply propagated the random sampling errors. Of course, this is only an approximate estimate, due to the small number statistics. The typical uncertainty in the distance modulus due to the statistical errors is about $0.5 \mathrm{mag}$ or a factor of $\sim 1.5$.

There is an error component associated with the differential reddening within the cluster. This error can be estimated from the width of the "decontaminated" cluster sequences on the color-magnitude diagrams. Discarding the outliers, which are most likely stars with near-infrared excess, the typical halfwidth of the cluster sequences is $\Delta\left(J-K_{\mathrm{S}}\right) \sim 0.5 \mathrm{mag}$, corresponding to $\mathrm{A}_{K}<0.5 \mathrm{mag}$. This is comparable to the uncertainties of our distance modulus due to the random errors discussed above.

Summarizing all uncertainties discussed above, we obtain $\sigma\left\{(\mathrm{m}-\mathrm{M})_{0}\right\} \sim 2.5 \mathrm{mag}$ or a factor of $\sim 10$. However, these calculations are made under the assumption that the 10th brightest star is indeed on the main sequence, and if it is not, this could introduce much larger errors into the distance estimate. 
Therefore, the distances derived from this method have to be treated with extreme caution.

\section{Discussion and summary}

We have presented deep near-infrared imaging with angular resolution superior to $2 \mathrm{MASS}$ which indicates that the identification of clusters on the 2MASS images alone is far from reliable. The 2MASS images suffer from large pixel size and poor seeing conditions that have made many bright single or double stars appear as compact clusters. Furthermore, there are a number of cluster candidates that consist of a few - usually fewer than a dozen bright stars, - that are not associated with concentrations of fainter stars. Here we refrain from classifying those as clusters. However, they may be remnants of clusters, i.e. groups of common proper motion that have been ejected from larger stellar clusters. There is also the possibility that they may be genuine "clusters" with extremely peculiar IMFs with high lower cut-off.

Seven of the 21 observed objects appear to be typical young clusters. Most of them show extended gas emission, suggesting the presence of OB stars. Whenever possible, we estimated the mass of the clusters or at least of the probable cluster members for which we have photometry. These estimates range from a few hundred to a few thousand solar masses. None of the objects appear to be comparable in appearance and total mass to the Arches - estimated to have a dynamical mass of $7 \times 10^{4} M_{\odot}$ (Figer et al. 1999a) - or the Quintuplet clusters - estimated $\sim 10^{4} M_{\odot}$ from an integration of the cluster IMF (Figer et al. 1999b).

A special word of caution is necessary when discussing the masses of the clusters. The photometry alone is not sufficient to derive the distances to the clusters, and we were forced to apply a technique based on assuming a spectral type for the 10th brightest cluster member. There is a number of uncertainties in this method, due to the age, foreground and background contamination, and even the variations in the cluster population due to small number statistics. Spectroscopic observations to derive the spectral types and distances would be much more accurate, but this is beyond the scope of this paper.

Acknowledgements. This publication makes use of data products from the Two Micron All Sky Survey, which is a joint project of the University of Massachusetts and the Infrared Processing and Analysis Center/California Institute of Technology, funded by the National Aeronautics and Space Administration and the National Science Foundation. This research has made use of the SIMBAD database, operated at CDS, Strasbourg, France. The authors gratefully acknowledge the comments by an anonymous referee.
J.B., D.M. and D.G. are supported by FONDAP Center for Astrophysics grant number 15010003.

\section{References}

Bertelli, G., Bressan, A., Chiosi, C., Fagotto, F., \& Nasi, E. 1994, A\&AS, 106, 275

Bica, E., Dutra, C. M., Soares, J., \& Badbuy, B. 2003a, A\&A, 397, 177

Bica, E., Dutra, C. M., Soares, J., \& Badbuy, B. 2003b, A\&A, 404, 223

Blum, R. D., \& Damineli, A. 1999, ApJ, 512, 237

Borissova, J., Pessev, P., Ivanov, V. D., et al. 2003, A\&A, 411, 83 (Paper II)

Carpenter, J. M. 2000, AJ, 120, 3139

Churchwell, E., Walmsley, C. M., \& Cesaroni, R. 1990, A\&AS, 83,119

Elmegreen, B. 1998, in Origins, ed. C. E. E. Woodward, J. M. Shull, \& H. A. Tronson, ASP Conf. Ser., 148, 150

Epchtein, N. 1997, in The Impact of Large Scale Near-IR Sky Surveys, ed. F. Garzon, et al. (Dordrecht: Kluwer), ASSL, 210, 15

Dutra, C. M., \& Bica, E. 2000, A\&A, 359, L9

Dutra, C. M., \& Bica, E. 2001, A\&A, 376, 434

Dutra, C. M., \& Bica, E. 2002, A\&A, 383, 631

Dutra, C. M., Bica, E., Soares, J., \& Badbuy, B. 2003a, A\&A, 400, 533

Dutra, C. M., Ortolani, S., Bica, E., et al. 2003b, A\&A, 408, 127

Figer, D., Kim, S., Morris, M., et al. 1999, ApJ, 525, 750

Figer, D. F., McLean, I. S., \& Morris, M. 1999, ApJ, 514, 202

Figer, D. F., Najarro, F., Gilmore, D., et al. 2002, ApJ, 581, 258

Hurt, R. L., Jarrett, T. H., Kirkpatrick, J. D., et al. 2000, AJ, 120, 1876

Glass, I. S., Catchpole, R. M., \& Whitelock, P. A. 1987, MNRAS, 227, 373

Ivanov, V. D., Borissova, J., Pessev, P., Ivanov, G. R., \& Kurtev, R. 2002, A\&A, 394, 1 (Paper I)

Kobulnicky, H., Monson, A., Buckalew, J., et al. 2005, AJ, 129, 239

Nagata, T., Hyland, A. R., Straw, S. M., Sato, S., \& Kawara, K. 1993, ApJ, 406, 501

Porras, A., Cruz-Gonzalez, I., \& Salas, L. 2000, A\&A, 361, 660

Reylé, C., \& Robin, A. C. 2002, A\&A, 384, 403

Salpeter, E. E. 1955, ApJ, 121, 161

Schmidt-Kaler, T. 1982, in Landolt-Borstein, New Series, Group VI, ed. K. Schaifers, \& H. H. Voigt (Berlin: Springer-Verlag), 2, 1

Sharpless, S. 1959, ApJS, 4, 257

Schultheis, M., Lann, A., Omont, A., Schuller, F., \& Ojha, D. K. 2003, A\&A, 405, 531

Skrutskie, M. F., et al. 1997, in The Impact of Large Scale Near-IR Sky Surveys, ed. F. Garzon, et al. (Dordrecht: Kluwer), ASSL, 210,25

Stetson, P. B. 1993, User's Manual for DAOPHOT II

Storm, K., Strom, S., \& Merrill, M. 1993, ApJ, 233

Walsh, A. J., Hyland, A. R., Robinson, G., \& Burton, M. G. 1997, MNRAS, 291, 261 